Introduction: Anatomical changes after surgery and fibrotic adhesions increase the organ laceration risk, in cluding that of the ureter, in recurrent cases and secondary operations. The aim of this study was to investigate the changes in the anatomical localisations of the ureters via computed tomography urography in patients undergoing rectal cancer surgery.

Material and methods: The study involved prospectively collected data on the changes of ureteral location preoperatively and postoperatively in patients with operated rectal cancer. Distances $(\mathrm{mm})$ of ureters determined midline in the computed tomography urogram phase.

Results: A total of 18 patients were included. The mean distances between the right $\left(R_{1}\right)$ and left $\left(L_{1}\right)$ ureters and the mid-vertebral line before the surgery were $30.9 \pm 5.4 \mathrm{~mm}$ and $34.5 \pm 9.9 \mathrm{~mm}$, respectively. The postoperative distances between them $(R$ and $L_{2}$ ) were $26.4 \pm 9.1 \mathrm{~mm}$ and 29.5 $\pm 9.9 \mathrm{~mm}$, respectively. The $R_{2}$ measurement showed that $83.3 \%(15 / 18)$ of the right ureters had deviated medially, whereas $16.7 \%(3 / 18)$ of them had deviated laterally. The $L_{2}$ measurements showed that $88.8 \%(16 / 18)$ of the left ureters had deviated medially, whereas $11.2 \%(2 / 18)$ of them had deviated laterally. The differences between the preoperative and postoperative measurements of the right and left ureter positions were $4.5 \pm 9.2 \mathrm{~mm}$ and $4.9 \pm 4.6 \mathrm{~mm}$, respectively, with the displacement in the left ureter being statistically significant $(p \leq 0.001)$. Conclusions: Rectal cancer surgery causes medially deviated changes in the positions of the ureters.

Key words: rectal cancer surgery, computed tomography urography, ureteral translocation.

Contemp Oncol (Pozn) 2019; 23 (3): 164-168 DOI: https://doi.org/10.5114/wo.2019.89244

\section{The effects of rectal cancer surgery on the anatomical localisation of ureters - a prospective observational study}

\author{
Ulaş Aday ${ }^{1}$, Ebubekir Gündeş ${ }^{2}$ Durmuş A. Çetin ${ }^{3}$, Hüseyin Çiyiltepe ${ }^{4}$, \\ Selçuk Gülmez ${ }^{5}$, Aziz S. Senger ${ }^{5}$, Betül A. Özdere ${ }^{6}$, Mustafa Duman ${ }^{5}$, Erdal Polat ${ }^{5}$
}

\begin{abstract}
${ }^{1}$ Department of Gastroenterological Surgery, Elazıg Training and Research Hospital, University of Health Sciences, Elazıg, Turkey

2Department of Gastroenterological Surgery, Gaziyaşargil Training and Research

Hospital, University of Health Sciences, Diyarbakir, Turkey

'Department of Gastroenterological Surgery, Sanliurfa Training and Research Hospital, Sanliurfa, Turkey

${ }^{4}$ Department of Gastroenterological Surgery, Fatih Sultan Mehmet Training and Research Hospital, Istanbul, Turkey

${ }^{5}$ Department of Gastroenterological Surgery, Kartal Koşuyolu Training and Research Hospital, University of Health Sciences, Istanbul, Turkey

${ }^{6}$ Department of Radiology, Kartal Koşuyolu Training and Research Hospital, University of Health Sciences, Istanbul, Turkey
\end{abstract}

\section{Introduction}

Colorectal malignancies occur frequently and are the fourth most common cause of malignancy-related mortality in the world. Approximately $1,096,000$ new cases of colon cancer are estimated to be diagnosed in 2018, while approximately 704,000 new cases of rectal cancer are expected worldwide. In addition, rectal cancer is the $10^{\text {th }}$ most deadly, with 310,000 deaths, representing 3.2\% of all cancer deaths worldwide [1]. During colorectal surgery, iatrogenic injuries to the urogenital system are well-known problems. In one large series, the risk of ureteral injury in colorectal surgery was reported as $0.24-5 \%$ [2, 3]. The risk factors for ureteral injury are malignancy, history of previous surgery, radiotherapy (RT) history, inflammatory bowel disease, diverticulitis, and massive intraabdominal bleeding. Abdominopelvic resection and sigmoidectomy in colorectal cancer surgery are the most common surgical procedures for ureteral injury. Moreover, the distal 1/3 of the ureter is the most frequent location of injury. Unfortunately, the anatomical changes after surgery and fibrotic adhesions increase the organ laceration risk in recurrent cases and secondary operations $[3,4]$.

The local recurrences in rectal cancer have decreased significantly with the standard use of neoadjuvant chemoradiotherapy (CRT) and total mesorectal excision (TME). Despite this, the local rectal cancer recurrence rate after curative surgery is $4-11 \%$. Performing re-resections in suitable patients who develop isolated local recurrence has been suggested because of an increase in the survival $[5,6]$. Most of the recurrences develop within the first two years; therefore, the current guidelines suggest biannual tomographic control during the first three years [7]. Anatomical anomalies are the main reasons for ureteral injuries during surgery. However, the use of computed tomography urography (CTU) has been increasing over the last two decades, and the use of this imaging method has made important contributions to the detection of urinary tract pathologies and anatomical variations [4, 8, 9].

There are no previous investigations in the literature evaluating the effects of rectal cancer surgery on the anatomical localisation of the ureters. Therefore, the aim of this study was to investigate the changes in the an- 
atomical localisations of the ureters via CTU in patients undergoing rectal cancer surgery.

\section{Material and methods}

This study was registered at clinicaltrial.gov (NCT03007667) after receiving approval from the local Ethics Committee. Verbal and written informed consent was obtained from the participants. The study period was between 1 November 2016 and 30 September 2017.

\section{Inclusion and exclusion criteria}

The study group consisted of patients $\geq 18$ years old, with adenocarcinoma located in the rectum. The inclusion criteria were as follows:

- American Society of Anaesthesiologists (ASA) score of I-III,

- normal renal function,

- no history of major abdominal surgery,

- no RT history in the pelvic region,

- rectal carcinoma suitable for curative surgery (those graded as T4 according to pelvic magnetic resonance imaging [MRI] were excluded),

- sphincter protective surgery with an open surgical procedure,

- surgical resection with a TME,

- no intraoperative ureteral injuries,

- no urogenital system congenital anomalies,

- no urogenital system surgical interventions,

- no anatomical deviations, such as scoliosis or fractures in the bony elements of the vertebrae or pelvis,

- no inflammatory bowel disease or diverticulitis,

- no allergies to contrast media,

- sufficient filling of contrast media in both of the ureters up to the bladder.

\section{CTU evaluation protocol}

The patients diagnosed with rectal cancer were evaluated via tomography to determine the presence of metastases, operability, and staging. When needed, a pelvic $M R I$ and endorectal ultrasound (ERUS) were performed to evaluate the local disease. The CT scans were conducted in the radiology department of our hospital under the supervision of a radiology specialist (BAÖ). The CTU was performed in the standard supine position with 64-slice, 1-mm sections using an Aquilion CT scanner (Toshiba Corporation, Otawara, Japan). Before imaging, a $1.5 \mathrm{ml} / \mathrm{kg}$ dose of iodised contrast media was infused at a rate of $3 \mathrm{ml} / \mathrm{min}$, following the intake of 1.5 I of water $2 \mathrm{~h}$ previously. After the contrast medium infusion, images were taken of the arterial phase at the $30^{\text {th }}$ second, portal venous phase at the $70^{\text {th }}$ second, late venous phase at the $120^{\text {th }}$ second, and excretory phase limited to the pelvic region at between the $8^{\text {th }}$ and $12^{\text {th }}$ minute $[8,9]$. Two anatomical lines were determined to measure the anatomical changes in the ureters. The first of these was a vertical line reaching the midline of the spinous processes of the vertebrae. The second line, sacral 1 and sacral vertebra 2 , was identified as the transverse line passing through the middle of the joint. The distances from the junction of the midvertical line with the transverse line to the ureters were measured. The right ureter distances to the midvertical line measured preoperatively and postoperatively were defined as $R_{1}$ and $R_{2}$, respectively. The left ureter distances to the junction measured preoperatively and postoperatively were defined as $L_{1}$ and $L_{2}$, respectively (Fig. 1).

The tumour localisation in the rectum was determined according to the distance to the dentate line as follows: 1-5 cm was lower, 6-10 cm was mid, and 11-15 cm was upper. All of the surgical procedures were done by the same team using an open technique and a standard TME. The anastomosis was performed using a circular stapler, and a drain was placed in the pelvic region. The duration between the two CT sessions was determined as the time between the preoperative CT scan and the one performed at the first CT control. The demographic properties, body mass index (BMI, $\left.\mathrm{kg} / \mathrm{m}^{2}\right)$, ASA score, and comorbid conditions of each of the patients were recorded. In addition, the application of neoadjuvant RT and/or postoperative chemotherapy was determined. Any postoperative complications that developed before or after 30 days following the procedure were defined as early and late complica-
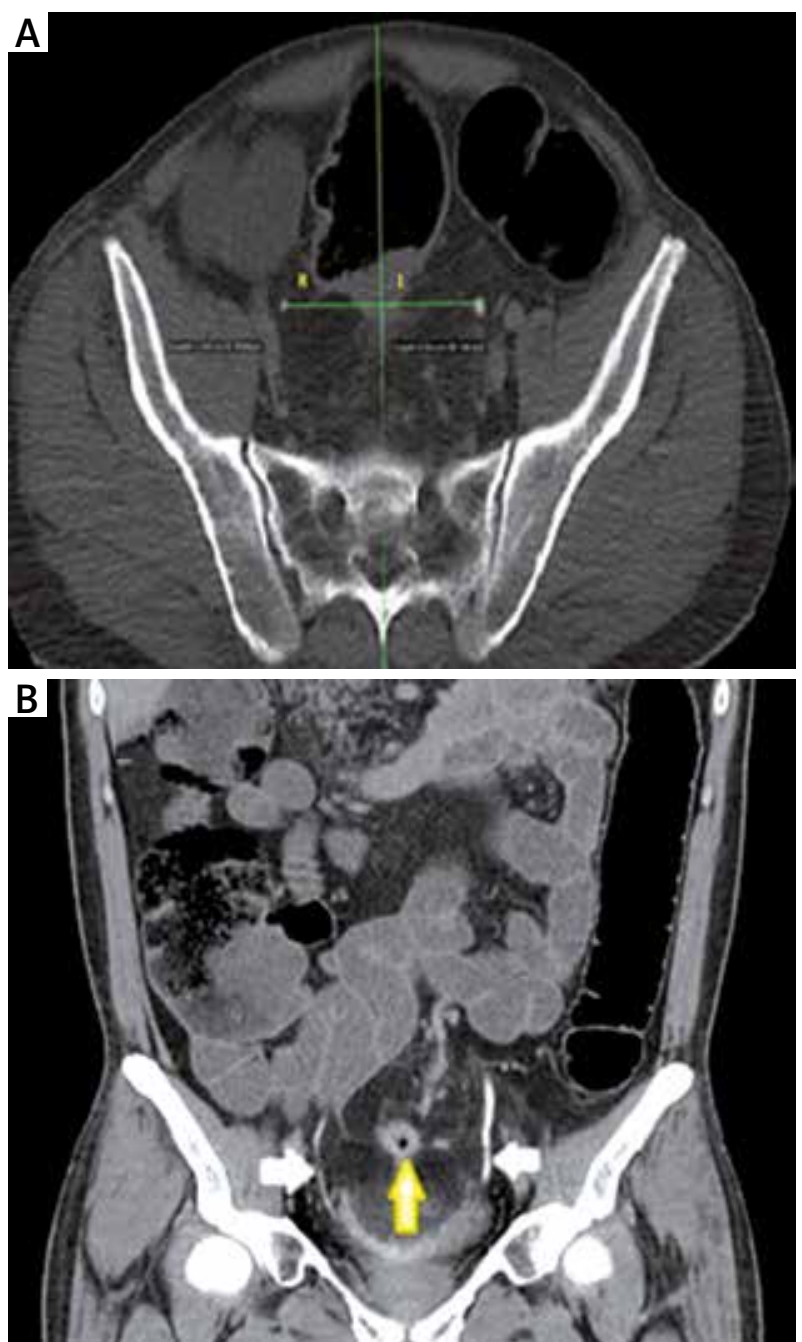

Fig. 1. A) The tomographic axial planar measurement of the right and left ureters. B) The white arrows show the ureter in the coronal tomography section, while the yellow arrow shows the tumour 
Table 1. Patient characteristics

\begin{tabular}{|c|c|}
\hline Variables & $n(\%)$ \\
\hline Age, mean \pm SD & $58.9 \pm 10.4$ \\
\hline $\begin{array}{l}\text { Sex } \\
\text { Male } \\
\text { Female }\end{array}$ & $\begin{array}{l}9(50) \\
9(50)\end{array}$ \\
\hline $\begin{array}{l}\text { Comorbidity } \\
\text { HT } \\
\text { DM } \\
\text { COPD } \\
\text { CAD }\end{array}$ & $\begin{array}{l}8(44.4) \\
4(22.2) \\
2(11.1) \\
2(11.1)\end{array}$ \\
\hline $\mathrm{BMI}\left(\mathrm{kg} / \mathrm{m}^{2}\right)$, mean $\pm \mathrm{SD}$ & $28.3 \pm 3.19$ \\
\hline $\begin{array}{c}\text { ASA } \\
\text { I } \\
\text { II } \\
\text { III }\end{array}$ & $\begin{array}{l}3(16.6) \\
8(44.4) \\
7(38.9)\end{array}$ \\
\hline $\begin{array}{l}\text { Tumour location } \\
\text { Low rectum } \\
\text { Middle rectum } \\
\text { Upper rectum }\end{array}$ & $\begin{array}{l}4(22.2) \\
6(33.3) \\
8(44.4)\end{array}$ \\
\hline $\begin{array}{l}\text { Preoperative radiotherapy } \\
\text { Yes } \\
\text { No }\end{array}$ & $\begin{array}{c}10(55.6) \\
8(44.4)\end{array}$ \\
\hline $\begin{array}{l}\text { Loop ileostomy } \\
\text { Yes } \\
\text { No }\end{array}$ & $\begin{array}{c}10(55.6) \\
8(44.4)\end{array}$ \\
\hline $\begin{array}{l}\text { Leak } \\
\text { Yes } \\
\text { No }\end{array}$ & $\begin{array}{c}2(11.1) \\
16(88.8)\end{array}$ \\
\hline $\begin{array}{l}\text { Postoperative chemotherapy } \\
\text { Yes } \\
\text { No }\end{array}$ & $\begin{array}{c}13(72.2) \\
5(27.8)\end{array}$ \\
\hline $\begin{array}{l}\text { T stage } \\
\text { O (pCR) } \\
\text { I } \\
\text { II } \\
\text { III } \\
\text { IV }\end{array}$ & $\begin{array}{c}1(5.6) \\
3(16.7) \\
2(11.1) \\
11(61.1) \\
1(5.6)\end{array}$ \\
\hline $\begin{array}{l}\text { Metastatic lymph nodes } \\
\text { N0 } \\
\text { N1 or N2 }\end{array}$ & $\begin{array}{l}11(61.1) \\
7(38.9)\end{array}$ \\
\hline Postoperative CTU time, months $\pm \mathrm{SD}$ & $7.7 \pm 1.5$ \\
\hline
\end{tabular}

tions, respectively. The pathological evaluations in this study were performed using the $7^{\text {th }}$ version of the American Joint Committee on Cancer's tumour, node, metastasis (TNM) staging system [10].

\section{Statistical analysis}

The Statistical Package for the Social Sciences version 22 (SPSS Inc., Chicago, IL, USA) was utilised for the biostatistical analysis. The patient data was stated as the standard deviation, minimum-maximum value, and percentage in the required fields. The Shapiro-Wilk's test was use for the numerical variables, and the significance of the changes in the anatomical localisations of the ureters were evaluated using a paired samples $t$ test. A $p$ value less than 0.05 was considered to be significant.

\section{Results}

Curative surgery was performed in 41 patients with rectal cancer in our clinic between 1 November 2016 and 30 September 2017. Twenty-three patients were excluded and 18 were included in this study. The mean age of the patients was $58.9 \pm 10.4$ years (range: $37-82$ years), and the male-to-female ratio was $1 / 1$. The comorbidity rates of the patients were as follows: hypertension $44.4 \%(n=8)$; diabetes mellitus, $22.2 \%(n=4)$; coronary artery disease, $11.1 \%(n=2)$; and chronic obstructive pulmonary disease, $11.1 \%(n=2)$. The mean BMI value was $28.3 \pm 3.19 \mathrm{~kg} / \mathrm{m}^{2}$. The ASA score rates were $16.6 \%(n=3)$ for ASA I, $44.4 \%$ $(n=8)$ for ASA II, and 38.9\% $(n=7)$ for ASA III. Ten $(55.5 \%)$ of the patients were given neoadjuvant RT. The mean duration between the two $\mathrm{CT}$ sessions was $7.7 \pm 1.5$ months (range: 6-12 months). The demographic and clinical data of the patients are summarised in Table 1.

The mean preoperative distance between the right ureter and the midvertebral line $\left(R_{1}\right)$ was $30.9 \pm 5.4 \mathrm{~mm}$, and the mean distance between the left ureter and the midvertebral line $\left(L_{1}\right)$ was $34.5 \pm 9.9 \mathrm{~mm}$. The postoperative distances of the right and left ureters $\left(R_{2}\right.$ and $L_{2}$, respectively) were $26.4 \pm 9.1 \mathrm{~mm}$ and $29.5 \pm 9.9 \mathrm{~mm}$, respectively. The postoperative measurements showed that $83.3 \%(15 / 18)$ of the right ureters shifted medially, while $16.7 \%$ (3/18) of them shifted laterally. The postoperative measurements of the left ureters showed that $88.8 \%(16 / 18)$ of them shifted medially and $11.2 \%$ (2/18) of them shifted laterally (Table 2$)$. The differences between the two measurements on the right and left were $4.5 \pm 92 \mathrm{~mm}$ and $4.9 \pm 4.6 \mathrm{~mm}$, respectively. The anatomical change amount in the left ureters was statistically significant $(p \leq 0.001)$. Figures 2 and 3 show the position changes of the ureters. During the early postoperative period, leakage developed in two patients whose right ureters shifted laterally. During the late postoperative period, one patient developed a stricture on the anastomosis line, and another developed a vaginal fistula from the anastomosis line. There were no complications in the third patient, whose right ureter shifted laterally.

Both patients whose left ureters deviated laterally also had lateral changes in their right ureters. Whereas there were neither early nor late complications in one of these patients, the other developed leakage and a stenosis in the late postoperative period. The patient who had a lateral deviation of the right ureter, but a medial deviation of the left ureter developed vaginal fistula.

The early postoperative complications consisted of anastomosis leaks in two patients and urinary retention, a wound infection, candidemia, ileus, and anastomosis bleeding in one patient each. All of these complications were treated medically and conservatively. Mortality did not develop in this study population.

\section{Discussion}

Studies investigating the effects of rectal carcinoma surgery with a TME on the anatomical localisation of the pel- 
Table 2. Results of preoperative and postoperative measurements

\begin{tabular}{|c|c|c|c|c|}
\hline Variables & $R_{1}$ & $R_{2}$ & $L_{1}$ & $L_{2}$ \\
\hline Distances of ureters, $\mathrm{mm} \pm \mathrm{SD}$ & $30.9 \pm 5.4$ & $26.4 \pm 9.1$ & $34.5 \pm 9.9$ & $29.5 \pm 9.9$ \\
\hline Displacement of the middle line, $n(\%)$ & \multicolumn{2}{|c|}{$15(83.3)$} & \multicolumn{2}{|c|}{$16(88.8)$} \\
\hline Lateral displacement, $n(\%)$ & \multicolumn{2}{|c|}{$3(16.7)$} & \multicolumn{2}{|c|}{$2(11.2)$} \\
\hline Difference between measurements, $\mathrm{mm} \pm \mathrm{SD}$ & \multicolumn{2}{|c|}{$4.5 \pm 9.2$} & \multicolumn{2}{|c|}{$4.9 \pm 4.6$} \\
\hline$p$-value & \multicolumn{2}{|c|}{0.052} & \multicolumn{2}{|c|}{$<0.001$} \\
\hline
\end{tabular}

Paired samples t-test was used; SD - standard deviation, $R_{1}$ - distance of preoperative right ureter, $R_{2}$ - distance of postoperative right ureter, $L_{1}$ - distance of preoperative left ureter, $L_{2}$ - distance of postoperative left ureter

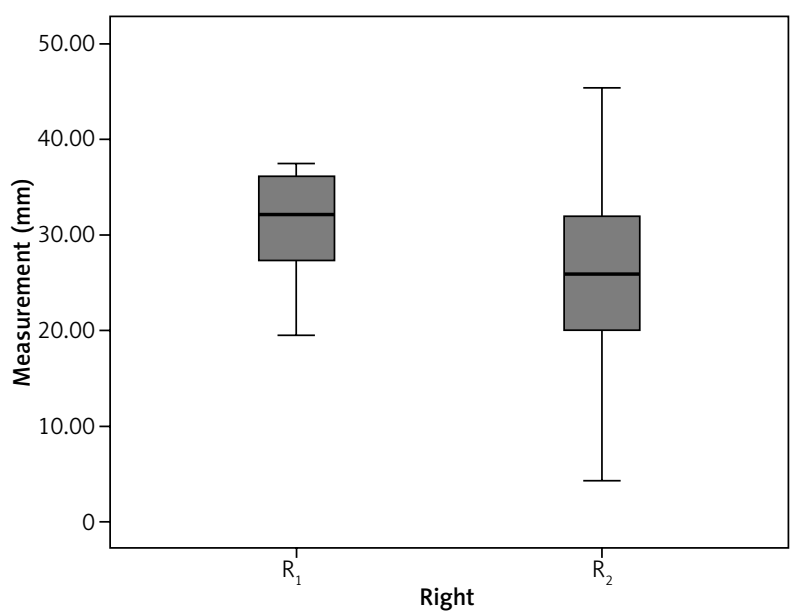

Fig. 2. Diagram showing the anatomical changes of the right ureter

vic ureters are limited. In our study, the CTU scans showed that the ureters moved closer to the midline in most of the patients. There were medial deviations in 15/18 (83.3\%) right ureters and 16/18 (88.8\%) left ureters, whereas a medial change in the position was frequent and more distant in the left ureters than in the right ones. Because the study population was limited, it is difficult to determine a valid reason for this difference. The inclusion of the sigmoid mesocolon during the TME procedure and the surgical dissection through the trace of the left ureter may have been the causes. One of the interesting results of this study was the presence of the same positional change in two of the three patients with lateral ureter deviations. The other patient with a lateral deviation of the right ureter developed a fistula from the anastomosis line to the posterolateral wall of the vagina during the second postoperative month. The medial change in the position of the left ureter was $11.8 \mathrm{~mm}$ in this patient, and the distance was significantly greater than the mean value. The inflammation due to the fistula may have contributed to the right deviation in the anatomical localisation. Although the dislocation in the right ureter was not statistically significant $(p=0.052)$, the dislocation in the left ureter was significant ( $p \leq 0.001)$.

Injuries to the urogenital system in colorectal surgery are likely complications because of the close anatomical vicinity. The ureter is the most frequently injured organ, and the complexity of the surgery increases the risk of damage. During colorectal surgery, the risk of injuring the

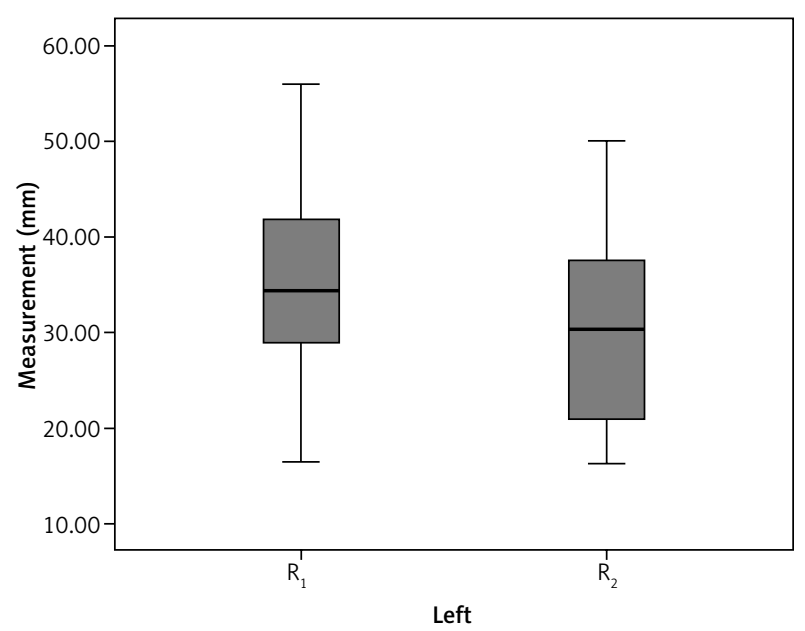

Fig. 3. Diagram showing the anatomical changes of the left ureter

ureter increases in certain circumstances, such as severe inflammation due to diverticulitis, inflammatory bowel disease, and pelvic infections. A locally advanced tumour stage, rectal carcinoma recurrence, and previous RT also increase the anatomical distortions [11, 12]. Ninety per cent of all iatrogenic injuries to the ureter develop in the distal part of the ureter [13]. A curative resection is possible via the development of new surgical techniques and procedures. However, fibrosis, scar tissue, and the loss of dissection planes due to previous surgery and RT increase the risk of injuring a neighbouring organ. The insertion of stents into the ureters is advised, if pelvic exenteration is not planned. Although it is under discussion, it is suggested that rate of ureteral injury is increased in laparoscopic colorectal surgery [2, 3, 14-17].

The use of CTU has been increasing over the last two decades, and it has made important contributions to the diagnosis of urinary tract pathologies and the detection of anatomical variations. CTU is the first-line diagnostic procedure in the evaluation of microscopic haematuria, and the excretory phase is the ideal phase to image the ureter and bladder [8, 9]. Moreover, sufficient distention in the distal parts of the ureters and bladder with contrast media filling is important to define the traces of the ureters. Therefore, it is recommended that the images be taken 8-12 min after the contrast media application to provide a suitable excretory phase. Attempts have been made to reduce the radiation dose by giving the contrast media in 
split boluses [18]. Four patients were excluded from this study because they were not provided with enough contrast media to fill the distal ureter.

This study did have some limitations. For example, it included a small study population, which complicated the definition of the factors influencing the dislocation of the ureters. In addition, the changes in the anatomical localisations were measured in two dimensions, while the changes in the anterior and posterior directions were not evaluated. Finally, a comparison related to the effects of the surgical procedures, such as abdominoperineal resection, low anterior resection, and open and laparoscopic techniques, could not be done.

\section{Conclusions}

Rectal carcinoma curative surgery including a TME may cause the medial dislocation of the ureters. Further larger case-control studies are necessary to define the factors causing these changes in anatomical localisations. CTU may be useful to prevent injury if surgery is to be performed for local recurrence. Surgeons should take into account the fact that the ureters move closer to the midline in patients with rectal carcinoma surgical histories when considering surgical treatments in recurrent cases.

\section{Acknowledgements}

We would like to thank Zeynep Özkan for his help in the statistical analysis of this article.

The authors declare no conflict of interest.

\section{References}

1. Rawla P, Sunkara T, Barsouk A. Epidemiology of colorectal cancer: incidence, mortality, survival, and risk factors. Gastroenterol Rev 2019; 14: 89-103.

2. Halabi WJ, Jafari MD, Nguyen VQ, Carmichael JC, Mills S, Pigazzi A, Stamos MJ. Ureteral injuries in colorectal surgery: an analysis of trends, outcomes, and risk factors over a 10-year period in the United States. Dis Colon Rectum 2014; 57: 179-186.

3. Eswara JR, Raup VT, Potretzke AM, Hunt SR, Brandes SB. Outcomes of iatrogenic genitourinary injuries during colorectal surgery. Urology 2015; 86: 1228-1234.

4. Delacroix SE Jr, Winters JC. Urinary tract injures: recognition and management. Clin Colon Rectal Surg 2010; 23: 104-112.

5. Troja A, El-Sourani N, Abdou A, Antolovic D, Raab HR. Surgical options for locally recurrent rectal cancer-review and update. Int J Colorectal Dis 2015; 30: 1157-1163.

6. van der Meij W, Rambouts AJM, Rütten H, Bremers AJ, de Wilt $\mathrm{JH}$. Treatment of locally reccurent rectal carcinoma in previously (chemo) irradiated patients: a review. Dis Colon Rectum 2016; 59: 148-156.

7. Watanabe T, Itabashi M, Shimada Y, et al. Japanese Society for Cancer of the Colon and Rectum (JSCCR) Guidelines 2014 for treatment of colorectal cancer. Int J Clin Oncol 2015; 20: 207-239.

8. Van Der Molen AJ, Cowan NC, Mueller-Lisse UG, Nolte-Ernsting CC, Takahashi S, Cohan RH; CT Urography Working Group of the European Society of Urogenital Radiology (ESUR). CT urography: definition, indications, and techniques. A guideline for clinical practice. Eur Radiol 2007; 18: 4-14.

9. Potenta SE, D’Agostino R, Sternberg KM, Tatsumi K, Perusse K. CT urography for evaluation of the ureter. Radiographics 2015; 35 : 709-726.
10. Stephan B, Edge DRB, Compton CC. AJCC Cancer Staging Manual (7 $7^{\text {th }}$ ed.). Springer-Verlag, New York 2010.

11. Fröber R. Surgical anatomy of the ureter. BJU Int 2007; 100: 949 965.

12. Althumairi AA, Efron JE. Genitourinary considerations in reoperative and complex colorectal surgery. Clin Colon Rectal Surg 2016; 29: 145-151.

13. Esparaz AM, Pearl JA, Herts BR, LeBlanc J, Kapoor B. Iatrogenic uri nary tract injuries: etiology, diagnosis, and management. Semin Intervent Radiol 2015; 32: 195-208.

14. Troja A, El-Sourani N, Abdou A, Antolovic D, Raab HR. Surgical options for locally recurrent rectal cancer-review and update. Int J Colorectal Dis 2015; 30: 1157-1163.

15. Harji DP, Griffiths B, McArthur DR, Sagar PM. Surgery for recurrent rectal cancer: higher and wider? Colorectal Dis 2013; 15: 139-145.

16. Abboudi H1, Ahmed K, Royle J, Khan MS, Dasgupta P, N'Dow J. Ureteric injury: a challenging condition to diagnose and manage. Nat Rev Urol 2013; 10: 108-115.

17. Andersen P, Andersen LM, Iversen LH. Iatrogenic ureteral injury in colorectal cancers surgery: a nationwide study comparing laparoscopic and open approaches. Surg Endosc 2015; 29: 1406-1412.

18. Silverman SG, Leyendecker JR, Amis ES. What is the current role of CT urography and MR urography in the evaluation of the urinary tract? Radiology 2009; 250: 309-323.

\section{Address for correspondence}

\section{Ulaş Aday}

Department of Gastroenterological Surgery

Elazıg Training and Research Hospital

University of Health Sciences

23100 Elazıg, Turkey

e-mail: ulasaday@gmail.com

Submitted: 10.04 .2019

Accepted: 21.06.2019 